\title{
On the Complexity of Computing the Diameter of a Polytope
}

\author{
Alan M. Frieze* Shang-Hua Teng ${ }^{\dagger}$
}

May 22, 2006

\begin{abstract}
In this paper, some results on the complexity of computing the combinatorial diameter of a polytope are presented. We show that it is $D^{P}$-hard to determine the diameter of a polytope specified by linear inequalities with integer data. Our result partially resolves a long-term open question.
\end{abstract}

\section{Introduction}

The basic idea of the simplex method for linear programming is to find a path from a vertex of the underlying polyhedron to an optimal one along edges. In graph-theoretic terms, the simplex method computes a path in graph $\Gamma(P)$, the 1-complex formed by the vertices and edges of the input polytope $P$, from an initial vertex to an optimal one. The efficiency of the simplex method is determined by the length of the path it computes. Therefore, the diameter of the graph $\Gamma(P)$ provides a natural lower bound for the simplex method.

Although, the diameter of polyhedral graphs has been studied intensively (see Klee 1974 and Larman 1970), tight bounds on the diameter in terms of the number of facets are still not known. Kalai (1991) gave the first a subexponential upper bound on the maximum diameter of $d$-polytopes with

*Department of Mathematics, Carnegie Mellon University, Pittsburgh, PA 15213, USA. Supported in part by an NSF Grant

${ }^{\dagger}$ School of Computer Science, Carnegie Mellon University, Pittsburgh, PA 15213, USA. Supported in part by an NSF Grant 
$n$ facets. Recently, Kalai and Kleitman (1992) further improved the upper bound to $n^{\log d+2}$.

In this paper, we study the complexity of computing the diameter of a polytope. On one hand, it is easy to see that the diameter of a polytope can be computed in $\Delta_{3}$, under the assumption that the diameter is bounded by a polynomial in the number of facets. On the other hand, we show that it is $D^{P}$-hard to determine the diameter of a polytope given by its facets, where $D^{P}$ is the following class of languages defined by Papadimitriou and Yannakakis (1984).

$$
D^{P}=\left\{L_{1} \cap L_{2}: L_{1} \in N P \& L_{2} \in C O-N P\right\} .
$$

Our result partially resolves a long-term open question.

\section{Definitions and problems}

Let $V(P)$ denote the set of all vertices of a polytope $P, E(P)$ the set of all edges of $P$, and $F(P)$ be the set of all facets of $P$.

Define $\Gamma(P)$ to be the 1-complex formed by the vertices and edges of a polytope $P$, i.e., $\Gamma(P)=(V(P), E(P))$.

The distance of two vertices $u$ and $v$ in a graph $G$, denoted by distance $_{G}(u, v)$, is the length of the shortest path between $u$ and $v$ in $G$. The radius of a vertex $v$ in $G$ is

$$
\operatorname{radius}_{G}(v)=\max \left\{\operatorname{distance}_{G}(v, u): u \in G\right\}
$$

and the diameter of a graph $G$ is

$$
\text { diameter }(G)=\max \left\{\operatorname{radius}_{G}(v): v \in G\right\} .
$$

The diameter of a polytope $P$ is defined to be the diameter of $\Gamma(P)$. We study the following computational problem:

COMPUTING DIAMETER: given $P=\{x: A x \leq b\}$, a set of $n$ half-spaces in $m$ dimensions, compute the diameter of $\Gamma(P)$.

The following are two related decision problems,

- DIAMETER: given a polytope $P=\{x: A x \leq b\}$ and an integer $k$, is $k$ the diameter of $\Gamma(P)$ ?

- RADIUS: given a polytope $P=\{x: A x \leq b\}$, a vertex $v$ of $P$, and an integer $k$, is $\operatorname{radius}_{\Gamma(P)}(v)=k$ ? 


\section{The NP-hardness of computing DIAME- TER}

When a graph has $N$ vertices, using Dijkstra's shortest path algorithm, the diameter can be computed in $O\left(N^{3}\right)$ time. However, in general, the number of vertices of a polytope may be $\Omega\left(n^{m / 2}\right)$. Only when the dimension is fixed, can breadth-first search be used to compute the diameter of a polytope in polynomial time.

In this section, we give a proof that DIAMETER is $N P$-hard. The idea of the proof will be used in the next section to show that DIAMETER is in fact $D^{P}$-hard. The reduction is from the following $N P$-complete problem (see Karp 1972 and Garey \& Johnson 1979).

EXACT PARTITION: given a finite set $A=\left\{s_{1}, \ldots, s_{2 m}\right\}$ of integers, is there a subset $A^{\prime} \subset A$ with $\left|A^{\prime}\right|=m$ and

$$
\sum_{s \in A^{\prime}} s=\sum_{t \in A-A^{\prime}} t ?
$$

\subsection{The basic reduction}

The basic idea is to show that for each instance $A$ of EXACT PARTITION, we can, in polynomial time, construct a polytope $P_{A}$ with a polynomial number of faces and an integer $k$ such that

$$
\text { diameter }\left(P_{A}\right)= \begin{cases}k & \text { if } A \text { has an exact partition, } \\ k-1 & \text { if } A \text { has no exact partition. }\end{cases}
$$

Note first that EXACT PARTITION with $A=\left\{s_{1}, \ldots, s_{2 m}\right\}$ is equivalent to an integer linear program of the following simple form ILP1 (see Korte \& Schrader 1981).

$$
\begin{aligned}
& \begin{array}{l}
\text { maximize } \\
\text { subject to }
\end{array} \\
& \qquad \begin{aligned}
\sum_{i=1}^{2 m} s_{i} x_{i} & \leq \frac{1}{2} S \\
\sum_{i=1}^{2 m} d_{i} x_{i} & \leq \frac{1}{2} \sum_{i=1}^{2 m} d_{i} \\
x_{i} & \in\{0,1\}
\end{aligned}
\end{aligned}
$$


where $S=\sum_{i=1}^{2 m} s_{i}, s_{\max }=\max _{i} s_{i}$, and $d_{i}=s_{\max }-s_{i}$.

Lemma 1 (Korte and Schrader) A has an exact partition iff ILP1 has an optimal solution of value $m$.

Note also that all coefficients in ILP1 are non-negative. Let $M=\left(\sum_{i=1}^{2 m} s_{i}\right)+$ 1. We modify ILP1 to the following integer linear program: ILP2

$$
\begin{aligned}
& \text { maximize } \quad \sum_{i=1}^{2 m} x_{i} \\
& \text { subject to } \\
& \begin{aligned}
\sum_{i=1}^{2 m}\left(M+s_{i}\right) x_{i} & \leq \frac{1}{2} S+m M+\epsilon \\
\sum_{i=1}^{2 m}\left(M+d_{i}\right) x_{i} & \leq \frac{1}{2} \sum_{i=1}^{2 m} d_{i}+m M+\epsilon \\
x_{i} & \in\{0,1\}
\end{aligned}
\end{aligned}
$$

Clearly, ILP1 has an optimal solution of value $m$ iff ILP2 has an optimal solution of value $m$. Further we chose $\epsilon=\frac{1}{2 M}$ in ILP2 so that the linear programming relaxation of ILP2 is non-degenerate.

Proposition 2 When $\epsilon=\frac{1}{2 M}$, the polytope defined by the linear programming relaxation of $I L P 2$ is non-degenerate.

Proof Suppose the polytope defined by the linear programming relaxation of ILP2 is degenerate, then there are $2 m+1$ inequalities that are satisfied with equality by a common point $x$. This implies that

$$
\left|\left\{i: x_{i} \in\{0,1\}\right\}\right| \geq 2 m-1 .
$$

Case 1: $x_{i} \in\{0,1\}, i=1,2, \ldots, 2 m$. In this case, one of the first two inequalities is tight which implies that $2 \epsilon$ is an integer, a contradiction.

Case 2: $\left|\left\{i: x_{i} \in\{0,1\}\right\}\right|=2 m-1$. Without loss of generality, assume $0<x_{1}<1$. Then,

$$
\begin{aligned}
& \sum_{i=1}^{2 m}\left(M+s_{i}\right) x_{i}=\frac{1}{2} S+m M+\epsilon \\
& \sum_{i=1}^{2 m}\left(M+d_{i}\right) x_{i}=\frac{1}{2} \sum_{i=1}^{2 m} d_{i}+m M+\epsilon
\end{aligned}
$$


After eliminating $x_{1}$, we see that there is an integer $c$, such that

$$
\epsilon=\frac{c}{2 d_{1}}
$$

which contradicts with our assumption that $\epsilon=\frac{1}{2 M}$, and therefore the proposition follows.

Proposition 3 The value of the optimal solution of ILP2 is either $m$ or $m-1$.

Proof This is because all $\vec{x}=\left(x_{1}, \ldots, x_{2 m}\right)$ with $\sum_{i=1}^{2 m} x_{i}=m-1$ are feasible solutions to ILP2 and no $\vec{x}$ with $\sum_{i=1}^{2 m} x_{i} \geq m+1$ is a feasible solution to ILP2.

Consequently,

Lemma 4 ILP2 has an optimal solution of value $m$ if $A$ has an exact partition, otherwise the optimal value is $m-1$.

By relaxing the integrality constraints in ILP2, we obtain a polytope $P_{A}^{\prime}$ defined to be the set of $\vec{x}$ satisfying

$$
\begin{aligned}
\sum_{i=1}^{2 m}\left(M+s_{i}\right) x_{i} & \leq \frac{1}{2} S+m M+\epsilon \\
\sum_{i=1}^{2 m}\left(M+d_{i}\right) x_{i} & \leq \frac{1}{2} \sum_{i=1}^{2 m} d_{i}+m M+\epsilon \\
0 \leq x_{i} & \leq 1
\end{aligned}
$$

Geometrically, $P_{A}^{\prime}$ is a polytope obtained from the unit $2 m$-cube by cutting it with two half-spaces with non-negative coefficients. We denote the associated boundary hyperplanes by $H S_{1}$ and $H S_{2}$ in the following discussion. Now $\overrightarrow{0}$, the origin of $2 m$-space, is a vertex of $P_{A}^{\prime}$, and the diameter of $P_{A}^{\prime}$ is bounded by $2 m+4$. This latter fact follows immediately from

\section{Lemma 5}

$$
\operatorname{radius}_{\Gamma\left(P_{A}^{\prime}\right)}(\overrightarrow{0})= \begin{cases}m+2 & \text { if } A \text { has an exact partition } \\ m+1 & \text { if } A \text { has no exact partition }\end{cases}
$$


Proof We first prove that $\operatorname{radius}_{\Gamma\left(P_{A}^{\prime}\right)}(\overrightarrow{0}) \leq m+2$, if $A$ has an exact partition, and $\operatorname{radius}_{\Gamma\left(P_{A}^{\prime}\right)}(\overrightarrow{0}) \leq m+1$ otherwise.

The set of vertices in $P_{A}^{\prime}$ can be partitioned into three subsets;

- $V_{0}$ : the set of all vertices lying neither on $H S_{1}$ nor $H S_{2}$;

- $V_{1}$ : the set of all vertices lying on one of $H S_{1}$ or $H S_{2}$, but not both, and

- $V_{2}$ : the set of all vertices lying on both $H S_{1}$ and $H S_{2}$.

Note that all components of a vertex in $V_{0}$ are either 0 or 1 . Since the linear system (2) is non-degenerate, all vertices in $V_{1}$ have exactly one noninteger co-ordinate and all vertices in $V_{2}$ have exactly two non-integer coordinates, and all other co-ordinates are either 0 or 1.

It follows from Proposition 3 , that the number of components of value 1 of a vertex of $P_{A}^{\prime}$ is bounded from above by $m$ if $A$ has an exact partition, and by $m-1$ otherwise.

Note first that the distance from $\overrightarrow{0}$ to each vertex $\vec{v}$ in $V_{0}$ is equal to the number of components of value 1 in $\vec{v}$, which is bounded from above by $m$ if $A$ has an exact partition, and by $m-1$ otherwise.

Consider a vertex $\vec{v}$ in $V_{i}$ for $i \in\{1,2\}$. Without loss of generality assume $\vec{v}$ is on $H S_{1}$ and $\vec{v}=\left(v_{1}, \ldots, v_{2 m}\right)$ with $v_{j}=1$ for $1 \leq j \leq l, v_{l+1}$ and $v_{l+i}$ non-integral, $i \in\{1,2\}$, and $v_{j}=0$ for $l+i<j \leq 2 m$. Note that $l \leq m$ if $A$ has an exact partition, and $l \leq m-1$ otherwise.

Let $\vec{v}^{\prime}=\left(v_{1}^{\prime}, \ldots, v_{2 m}^{\prime}\right)$ with $v_{j}^{\prime}=1$ for $1 \leq j \leq l+i-1$ and $v_{j}^{\prime}=0$ for $l+1 \leq j \leq 2 m$. Using non-degeneracy, we see that $\vec{v}^{\prime} \in V\left(P_{A}^{\prime}\right) \cap V_{i-1}$. Note that $\left(\vec{v}, \vec{v}^{\prime}\right) \in E\left(P_{A}^{\prime}\right)$ because we can obtain the basic feasible solution associated with $\vec{v}$ from the one associated with $\vec{v}^{\prime}$ by replacing $x_{l+i}=0$ by $H S_{i}$. Consequently. the distance from $\overrightarrow{0}$ to a vertex $\vec{v}$ in $V_{i}$ is bounded by $l+i$ (a two step induction.)

We now prove that $\operatorname{radius}_{\Gamma\left(P_{A}^{\prime}\right)}(\overrightarrow{0}) \geq m+2$, if $A$ has an exact partition, and $\operatorname{radius}_{\Gamma\left(P_{A}^{\prime}\right)}(\overrightarrow{0}) \geq m+1$ otherwise.

By adding slack variables, the linear system (2) takes the form 


$$
\begin{aligned}
\sum_{i=1}^{2 m}\left(M+s_{i}\right) x_{i}+z_{1} & =\frac{1}{2} S+m M+\epsilon \\
\sum_{i=1}^{2 m}\left(M+d_{i}\right) x_{i}+z_{2} & =\frac{1}{2} \sum_{i=1}^{2 m} d_{i}+m M+\epsilon \\
x_{i}+y_{i} & =1 \\
x_{i}, y_{i}, z_{k} & \geq 0
\end{aligned}
$$

Note that, the set of basic variables associated with the vertex $\overrightarrow{0}$ is $B V_{1}=$ $\left\{y_{1}, \ldots, y_{2 m}, z_{1}, z_{2}\right\}$.

First of all assume that $A$ has an exact partition and without loss of generality, assume $\left\{s_{1}, \ldots, s_{m}\right\}$ and $\left\{s_{m+1}, \ldots, s_{2 m}\right\}$ is one. Since the linear system (2), and hence (3) is non-degenerate, there is a basic feasible solution of the following form:

$$
\begin{aligned}
\left(x_{i}=1\right) \&\left(y_{i}=0\right) & 1 \leq i \leq m \\
\left(x_{m+1}=\zeta_{1}\right) \&\left(y_{m+1}=1-\zeta_{1}\right) \&\left(z_{m+1}=0\right) & \\
\left(x_{m+2}=\zeta_{2}\right) \&\left(y_{m+2}=1-\zeta_{2}\right) \&\left(z_{m+2}=0\right) & \\
\left(x_{j}=0\right) \&\left(y_{j}=1\right) & m+3 \leq j \leq 2 m
\end{aligned}
$$

where $0<\zeta_{1}, \zeta_{2}<1$.

The vertex associated with the above basic feasible solution is

$$
\vec{v}=\left(1, \ldots, 1, \zeta_{1}, \zeta_{2}, 0, \ldots 0\right),
$$

and the associated basic variables are

$$
B V_{2}=\left\{x_{1}, \ldots, x_{m}, x_{m+1}, x_{m+2}, y_{m+1}, \ldots, y_{2 m}\right\} .
$$

¿From $\left|B V_{1}-B V_{2}\right| \geq m+2$ it follows that distance $(\overrightarrow{0}, \vec{v}) \geq m+2$ and hence $\operatorname{radius}_{\Gamma\left(P_{A}^{\prime}\right)}(\overrightarrow{0}) \geq m+2$.

Similarly, if $A$ does not have an exact partition then we can show $\operatorname{radius}_{\Gamma\left(P_{A}^{\prime}\right)}(\overrightarrow{0})$ $\geq m+1$, by considering the basic feasible solution $\left(1, \ldots, 1, \zeta_{1}, \zeta_{2}, 0, \ldots 0\right)$ where there are $m-11$ 's.

Consequently,

Theorem 6 RADIUS is NP-hard. 

(1).

We now show how to construct the polytope $P_{A}$ from $P_{A}^{\prime}$, which satisfies

¿From the definition of $P_{A}^{\prime}$, we have for all $i: 1 \leq i \leq 2 m, \vec{w}_{i}=$ $\left(\mu_{1}, \ldots, \mu_{2 m}\right)$, with $\mu_{i}=1$ and $\mu_{j}=0$, for all $j \neq i: 1 \leq j \leq 2 m$, are vertices of $P_{A}^{\prime}$. Moreover, they are exactly the set of all neighbors of $\overrightarrow{0}$ in $\Gamma\left(P_{A}^{\prime}\right)$.

By adding the constraint $H_{0}=\left\{x: \sum_{i=1}^{2 m} x_{i} \geq 1\right\}$ to $P_{A}^{\prime}$, we obtain a new polytope $P_{A}^{\prime \prime}$ in which $F_{0}=H_{0} \cap P_{A}^{\prime}$ is a face. $F_{0}$ is a $(2 m-1)$-simplex with the set of vertices $\left\{\vec{w}_{1}, \ldots, \vec{w}_{2 m}\right\}$.

Now the idea is to construct a polytope $P_{2 m}\left(F_{0}, m+6\right)$, a stack of simplices with base $F_{0}$, which has the following properties

1. there is a vertex $\vec{o}$ in $P_{2 m}\left(F_{0}, m+6\right)$ such that the distance between $\vec{o}$ and any vertex in $F_{0}$ is $m+6$.

2. $P_{A}=P_{A}^{\prime \prime} \cup P_{2 m}\left(F_{0}, m+6\right)$ forms a polytope.

First, we give the construction of $P_{2 m}\left(F_{0}, m+6\right)$. Then, we shall show that

$$
\operatorname{diameter}\left(P_{A}\right)=\operatorname{radius}_{\Gamma\left(P_{A}\right)}(\vec{o})=\operatorname{radius}_{\Gamma\left(P_{A}^{\prime}\right)}(\overrightarrow{0})+m+5 \text {. }
$$

In the following procedure, let $\Delta=\sqrt{1 / 2 m}$, the distance from $\overrightarrow{0}$ to the hyperplane $\sum_{i} x_{i}=1$ and the symbol $\approx$ denotes the rational approximation with a predefined precision.

Procedure to Define $P_{2 m}\left(F_{0}, k\right)$ :

1. $P_{2 m}\left(F_{0}, 0\right)$ is defined to be the simplex with the set of vertices $\left\{\vec{w}_{1}, \ldots, \vec{w}_{2 m}\right\} \cup$ $\left\{\vec{o}_{0}\right\}$, where $\vec{o}_{0}=\overrightarrow{0}$;

2. for all $k>0, P_{2 m}\left(F_{0}, k\right)$ can be constructed from $P_{2 m}\left(F_{0}, k-1\right)$ as follows:

(a) $d_{k}=\frac{1}{2} \Delta+\frac{1}{2^{k+1}} \Delta$;

(b) $c_{k} \approx \sqrt{2 m} d_{k}$;

(c) $d_{k}^{\prime}=\frac{1}{2} \Delta-\frac{1}{2^{k+1}} \Delta$;

(d) Let $H_{k}=\left\{x: \sum_{i=1}^{2 m} x_{i} \geq c_{k}\right\}$;

(e) Let $\vec{o}_{k}=\left(\alpha_{k}, \ldots, \alpha_{k}\right)$, where $\alpha_{k} \approx \frac{d_{k}^{\prime}}{\sqrt{2 m}}$; 
(f) Let polytope $Q_{k}=P_{2 m}\left(F_{0}, k-1\right) \cap H_{k}$.

(g) Let polytope $P_{2 m}\left(F_{0}, k\right)$ be the convex hull of $\left\{\vec{o}_{k}\right\} \cup V\left(Q_{k}\right)$.

Note that $\vec{o}_{k}$ in the above procedure is the point on the ray $\left\{x_{i}=x_{j}: 1 \leq\right.$ $i, j \leq 2 m\}$, whose distance from $\overrightarrow{0}$ is $d_{k}^{\prime}$, and $H_{k}$ is the hyperplane parallel to $H_{0}$, whose distance from $\overrightarrow{0}$ is $d_{k}$.

Lemma 7 for all $k \geq 0$,

1. The polytope $P_{2 m}\left(F_{0}, k\right)$ has $2(k+1) m+1$ vertices and $2(k+1) m+1$ faces;

2. $P_{A}^{\prime \prime} \cup P_{2 m}\left(F_{0}, k\right)$ forms a polytope;

3. All coefficients in the new faces have size bounded polynomially in $m$ and $k$.

Proof We prove the theorem by induction on $k$.

Clearly, the lemma and the following statements are true when $k=0$.

1. $\vec{o}_{k}$ is a vertex of $P_{2 m}\left(F_{0}, k\right)$ and is the intersection of exactly $2 m$ faces;

2. All neighbors of $\vec{o}_{k}$ in $P_{2 m}\left(F_{0}, k\right)$ are on the hyperplane defined by $H_{k}$.

Assume the Lemma and the above statements are true for $k-1$. We now prove that they are true for $k$.

Note that the hyperplane defined by $H_{k}$ is parallel to the hyperplane defined by $H_{k-1}$ and $H_{k}$ separates $\vec{o}_{k-1}$ and $\vec{o}_{k}$ from $Q_{k}$. Moreover, $V\left(P_{2 m}\left(F_{0}, k-\right.\right.$ 1)) $-\left\{\vec{o}_{k-1}\right\} \subset V\left(Q_{k}\right)$.

Applying the induction hypotheses, we see that $\vec{o}_{k-1}$ is a vertex of $P_{2 m}\left(F_{0}, k-\right.$ 1) and is the intersection of exactly $2 m$ faces and all neighbors of $\vec{o}_{k}$ in $P_{2 m}\left(F_{0}, k-1\right)$ are on the hyperplane defined by $H_{k-1}$. Therefore, $H_{k} \cap Q_{k}$ contains exactly $2 m$ vertices of $Q_{k}$, and those $2 m$ vertices are all the new vertices introduced in $Q_{k}$, which do not belong to $V\left(P_{2 m}\left(F_{0}, k-1\right)\right)$, and hence,

$$
\left|F\left(Q_{k}\right)\right|=\left|F\left(P_{2 m}\left(F_{0}, k-1\right)\right)\right|+1 .
$$

Since, $\vec{o}_{k} \in$ int $P_{2 m}\left(F_{0}, k-1\right)$ and the hyperplane defined by $H_{k}$, which contains $2 m$ vertices of $Q_{k}$, separates $\vec{o}_{k}$ from $Q_{k}, P_{A}^{\prime \prime} \cup P_{2 m}\left(F_{0}, k\right)$ forms a polytope, and $\vec{o}_{k}$ is a vertex of $P_{2 m}\left(F_{0}, k\right)$ and is the intersection of $2 m$ faces 
of $P_{2 m}\left(F_{0}, k\right)$ and all neighbors of $\vec{o}_{k}$ in $P_{2 m}\left(F_{0}, k\right)$ are on the hyperplane $H_{k}$. Moreover,

$$
V\left(P_{2 m}\left(F_{0}, k\right)\right)=V\left(Q_{k}\right) \cup\left\{\vec{o}_{k}\right\}
$$

Therefore,

$$
\left|V\left(P_{2 m}\left(F_{0}, k\right)\right)\right|=\left|V\left(P_{2 m}\left(F_{0}, k-1\right)\right)\right|+2 m=2(k+1) m+1 .
$$

Similarly,

$$
\left|F\left(P_{2 m}\left(F_{0}, k\right)\right)\right|=\left|F\left(P_{2 m}\left(F_{0}, k-1\right)\right)\right|+2 m=2(k+1) m+1 .
$$

Note also, all coefficients in the new face have size bounded polynomially in $m$ and $k$.

\section{Lemma 8}

$$
\operatorname{diameter}\left(P_{A}\right)=\operatorname{radius}_{\Gamma\left(P_{A}^{\prime}\right)}(\overrightarrow{0})+m+5
$$

Proof ¿From the construction of $P_{A}$, we have for all vertices $\vec{v} \in$ $V\left(P_{A}\right)-V\left(P_{A}^{\prime}\right)$, distance $(\vec{o}, \vec{v}) \leq m+5$, and distance $\left(\vec{o}, \vec{w}_{i}\right)=m+6(1 \leq i \leq$ $2 m)$. Thus, for all $\vec{u} \in V\left(P_{A}^{\prime}\right)$, distance $_{P_{A}}(\vec{o}, \vec{u})=$ distance $_{P_{A}^{\prime}}(\overrightarrow{0}, \vec{u})+m+5$. Therefore,

$$
\operatorname{radius}_{\Gamma\left(P_{A}\right)}(\vec{o})=\operatorname{radius}_{\Gamma\left(P_{A}^{\prime}\right)}(\overrightarrow{0})+m+5 \geq 2 m+6 .
$$

We now prove that diameter $\left(P_{A}\right)=\operatorname{radius}_{\Gamma\left(P_{A}\right)}(\vec{o})$.

This is true because for all pairs of vertices in $P_{A}^{\prime}$, their distance in $P_{A}$ is no more than their distance in $P_{A}^{\prime}$, which is bounded by $2 m+4$; and for all vertices in $V\left(P_{A}\right)-V\left(P_{A}^{\prime}\right), \vec{o}$ is the vertex with the largest radius.

Theorem 9 DIAMETER is NP-hard.

Proof The theorem is a simple consequence of Lemmas 5 and 8 . 


\section{Polytope products and $D^{P}$-hardness}

Let $P_{1}$ and $P_{2}$ be two polytopes in, respectively, $m_{1}$ and $m_{2}$ space. $P_{1} \odot P_{2}$, the product of polytopes $P_{1}$ and $P_{2}$, is a polytope in $m_{1}+m_{2}$ space, such that $P_{1} \odot P_{2}=\left\{\left(x_{1}, \ldots, x_{m_{1}}, y_{1}, \ldots, y_{m_{2}}\right) \mid\left(x_{1}, \ldots, x_{m_{1}}\right) \in P_{1} \&\left(y_{1}, \ldots, y_{m_{2}}\right) \in P_{2}\right\}$. Note that $\odot$ is associative.

Algebraicly, if $P_{1}=\left\{x: A_{1} x \leq b_{1}\right\}$ and $P_{2}=\left\{y: A_{2} y \leq b_{2}\right\}$ then

$$
P_{1} \odot P_{2}=\left\{(x, y): A_{1} x \leq b_{1}, A_{2} x \leq b_{2}\right\} .
$$

Therefore, we have $f\left(P_{1} \odot P_{2}\right)=f\left(P_{1}\right)+f\left(P_{2}\right)$, where $f(P)$ denotes the number of faces of polytope $P$.

We now show how $\Gamma\left(P_{1} \odot P_{2}\right)$ is defined in term of $\Gamma\left(P_{1}\right)$ and $\Gamma\left(P_{2}\right)$.

The product of two graphs $G\left(V_{1}, E_{2}\right)$ and $G\left(V_{2}, E_{2}\right)$, denoted by $G_{1} \odot G_{2}$, is a new graph $G(V, E)$ with $V=V_{1} \times V_{2}$ and

$E=\left\{\left(\left(u_{1}, v_{1}\right),\left(u_{2}, v_{2}\right)\right):\left(u_{1}, u_{2}\right) \in E_{1} \& v_{1}=v_{2}\right.$ or $\left.\mathrm{u}_{1}=\mathrm{u}_{2} \&\left(\mathrm{v}_{1}, \mathrm{v}_{2}\right) \in \mathrm{E}_{2}\right\}$.

\section{Proposition 10}

$$
\Gamma\left(P_{1} \odot P_{2}\right)=\Gamma\left(P_{1}\right) \odot \Gamma\left(P_{2}\right)
$$

\section{Proposition 11}

$$
\operatorname{Diameter}\left(G_{1} \odot G_{2}\right)=\operatorname{Diameter}\left(G_{1}\right)+\operatorname{Diameter}\left(G_{2}\right) \text {. }
$$

Let PARTITON-UNPARTITON be the problem of "given $\left(A_{1}, A_{2}\right)$, where $A_{1}=\left\{s_{1}, \ldots, s_{2 n_{1}}\right\}$ and $A_{2}=\left\{s_{1}^{\prime}, \ldots, s_{2 n_{2}}^{\prime}\right\}$, does $A_{1}$ have an exact partition, while $A_{2}$ does not?".

Lemma 12 PARTITION-UNPARTITION is complete for $D^{P}$.

Proof Clearly, PARTITION-UNPARTITION is in $D^{P}$. To prove it is complete, we see that from any instance $x$ of a problem in $D^{P}$, we can construct two sets $A_{1}$ and $A_{2}$, one for the $N P$-predicate of $A$ and one for the co-NP one. 
Theorem 13 DIAMETER is hard for $D^{P}$.

Proof We reduce PARTITION-UNPARTITION to DIAMETER. Given $\left(A_{1}, A_{2}\right)$, we construct two polytopes $P_{1}$ and $P_{2}$, respectively, for $A_{1}$ and $A_{2}$, such that $A_{i}$ has an exact partition iff DIAMETER $\left(\Gamma\left(P_{i}\right)\right)=k_{i}$, and has no exact partition iff DIAMETER $\left(\Gamma\left(P_{i}\right)\right)=k_{i}-1$, where $k_{1} \neq k_{2}$. Let $P=P_{1} \odot P_{1} \odot P_{2}$. It is easy to see that $\left(A_{1}, A_{2}\right) \in$ PARTITIONUNPARTITION iff DIAMETER $(P)=2 k_{1}+k_{2}-1$.

Similarly we can prove,

Theorem 14 RADIUS is hard for $D^{P}$.

\section{Upper bound for DIAMETER}

Consider the following decision problem:

DIAMETER-DECISION: given $P=\{A x \leq b\}$ and $k \in \mathcal{N}$, is diameter $G(P) \leq k$ ?

Lemma 15 The problem DIAMETER-DECISION is in $\Pi_{2}$ under the assumption that the diameters of polytopes are polynomially bounded by the number of faces.

Proof The lemma follows from the facts that in polynomial time, we can decide whether a point is a vertex of a polytope and whether a pair of vertices is an edge of a polytope.

Using binary search and the $\Pi_{2}$ oracle for DIAMETER-DECISION, we can show that both DIAMETER and RADIUS are in $\Delta_{3}$ (under the polynomial assumption).

\section{Open questions}

1. Is DIAMETER in the polynomial time hierarchy (see Stockmeyer 1977) (without any assumption)?

2. Is DIAMETER complete for $\Delta_{3}$ and the DIAMETER-DECISION complete for $\Pi_{2}$ ? 
3. Can we approximate the diameters of polytopes in random polynomial time?

4. Can we improve the straightforward method for COMPUTING DIAMETER (especially for fixed dimension)?

Acknowledgement The research of Alan Frieze was supported in part by National Science Foundation Grant CCR-089-00112. The research of Shang-Hua Teng was supported in part by NSF Grant CCR-9016641. His current address is: Department of Mathematics and Laboratory for Computer Science, Massachusetts Institute of Technology, Cambridge, MA 02139.

\section{References}

[Garey \& Johnson 1979] M. R. Garey And D. S. Johnson, Computers and Intractability: A Guide to the Theory of NP-completeness, Freeman, San Francisco, 1979.

[Karp 1972] R. M. KARP, Reducibility among combinatorial problems, in Complexity of Computer Computation, (R. E. Miller and Thatcher, Eds.), pages 85-103. Plenum, New York, 1972.

[Kalai 1991] G. KALAI, Subexponential Bound for the $d$-step Problem, manuscript, IBM Almaden Research Center, 1991.

[Kalai \& Kleitman 1992] G. Kalai And D.J. Kleitman. A quasi-polynomial bound for diameter of graphs of polyhedra, (Bull. of the Amer. Math Soc.), 26(2) pp315-316, April, 1992.

[Klee 1974] V. KLEE, Polytope pairs and their relationship to linear programming, Acta Mathematica, 133(2):1-25, Octobre 1974.

[Korte \& Schrader 1981] B. Korte And R. Schrader, On the existence of fast approximating scheme, Nonlinear Programming 4, pp415-437, 1981.

[Larman 1970] D. G. Larman, Path on polytopes, Proc. London Math. Soc., $30: 161-178,1970$.

[Papadimitriou \& Yannakakis 1984] C. H. PApadimitriou And M. YanNAKAKIS, The complexity of facets, JCSS, 28:244-259, 1984. 
[Stockmeyer 1977] L. J. StockmeYeR, The polynomial-time hierarchy, Theoretical Computer Science, 3:1-22, 1977. 